\title{
Laser 3D-modeling in research of molecular features of skin lymphatic vessels in the patients with urticaria pigmentosa
}

\author{
Svetlana Viktorovna Michurina \\ Group of experimental \\ pharmacology \\ RICEL - Branch of ICG SB RAS \\ Novosibirsk, Russia \\ s.michurina@ngs.ru \\ Irina Yurievna Ishchenko \\ Group of experimental \\ pharmacology \\ RICEL - Branch of ICG SB RAS \\ Novosibirsk, Russia \\ irenisch@mail.ru
Konenkov Vladimir Iosifovich
Laboratory of clinical immunogenetics
Novosibirsk, Russia
vikonenkov@gmail.com \\ RICEL - Branch of ICG SB RAS
}

\author{
Svechnikova Natalia Nikolaevna \\ Laboratory of clinical \\ immunogenetics \\ RICEL - Branch of ICG SB RAS \\ Novosibirsk, Russia \\ n.svechnikova@ngs.ru
}

\author{
Sergey Alekseevich Arkhipov \\ Group of experimental \\ pharmacology \\ RICEL - Branch of ICG SB RAS \\ Novosibirsk, Russia \\ arhipowsergei@yandex.ru
}

\author{
Andrey Yurievich Letyagin \\ Laboratory of pharmaceutical \\ technologies \\ RICEL - Branch of ICG SB RAS \\ Novosibirsk, Russia \\ letyagin-andrey@yandex.ru
}

\author{
Solovyova Anastasia Olegovna \\ Laboratory of pharmacological active \\ compounds \\ RICEL - Branch of ICG SB RAS \\ Novosibirsk, Russia \\ solovevaao@gmail.com
}

\begin{abstract}
Congestion in the blood and lymphatic vessels is detected in the papillary and reticular layers in the skin dermis of patients with pigment urticarial. This promotes the development of microcirculatory disorders and the formation of perivascular infiltrates. LYVE-1 positive staining was detected not only in the endothelium of the lymphatic vessels (both in the skin papillary layer and in the deeper dermis layers), but also in the prickle cells of the stratum spinosum and (less pronounced) in the cells of the stratum basal in the epidermis. Our 3D modeling of the LYVE-1 marker expression in skin epidermis suggests that mainly the prickle cells of the epithelium stratum spinosum and to a lesser extent the cells of the epithelium basal layer in patients with cutaneous mastocytosis participate in the lymphatic transport of hyaluronan and the spaces between these cells can be considered as prelimfatiks of the skin epidermis.
\end{abstract}

Key words - urticaria pigmentosa, LYVE-1, prickle cells of the stratum spinosum

\section{Introduction}

About $85 \%$ of adult patients with mastocytosis have skin lesions (urticaria pigmentosa). The disease is classified as a rare dermatosis, the pathogenesis of which has not been studied enough. Along with comprehensive studies of the state of mast cells in the development of clinical manifestations of the disease, the lymphatic system of the human skin remains unexplored. This can be explained by the complexity of methods for studying the morphology of small lymphatic vessels. However, researchers often overlook the skin lymphatic vessels, since they are small slit-like structures above the hair follicles, resembling artifact-like separation of collagen bundles [1]. The structure of the lymphatic system of the skin was first described in Russia by D. A. Zhdanov in 1952 [2]. A little later, the skin lymphatic vessels were determined using electron microscopy. Selective markers for lymphatic endothelium were until recently rare or unavailable. Currently, lymphatic vessels are easily detected by the immunohistochemical method using LYVE-1, which is a cell surface receptor for lymphatic endothelial cells [3].

\section{Materials and Methods}

The paper presents clinical observations of 2 patients with a cutaneous form of mastocytosis, histological examination, immunohistochemical study. For histological examination, skin samples were fixed in 10\% buffered formalin in 48 hours, dehydrated in a series of alcohols with increasing concentrations, enclosed in histomix; sections were obtained by the microtome LEICA RM2155. Immunohistochemical detection of the LYVE-1 marker was performed in sections with a $3 \mu \mathrm{m}$ thickness using the indirect $\mathrm{ABC}$-peroxidase method using the VECTASTAIN Universal ABC-Peroxidase Kit (PK-7200, Vector Laboratories, USA). Endogenous peroxidase blocking was performed by incubation of sections in $0.3 \% \mathrm{H}_{2} \mathrm{O}_{2}$ solution for $10 \mathrm{~min}$, then sections were incubated with normal blocking non-immune horse serum for $20 \mathrm{~min}$. Next, the sections were incubated for an hour at room temperature with antibodies to LYVE-1 (Isotype: Rabbit polyclonal, bs-1311R; Bioss) using the final dilution of 5 $\mu \mathrm{g} / \mathrm{ml}$, then they were washed in 3 shifts of phosphate buffer (FB) for $3 \mathrm{~min}$ and were incubated for 30 minutes at room temperature with the second biotinylated antibodies followed by washing in 3 shifts of FB for 5 minutes. Incubation with the $\mathrm{ABC}$-peroxidase complex was carried out at room temperature for $30 \mathrm{~min}$, the sections were washed in 3 shifts of FB for 5 minutes. At the last stage immunohistochemical staining of the sections was performed in the chromogenic substrate (ImmPACT DAB, SK-4105, Vector Laboratories, США). During all immunohistochemical reactions, "negative controls" were carried out, replacing incubation with primary antibodies by incubation with non-immune sera of the corresponding animal (rabbit serum). Digital images of human skin preparations were taken with the AxioImager.M2 Zeiss microscope (Axiocam 305 Color camera). 3 D modeling of 
skin prelimphatics was performed using the laser holographic confocal microscope Nanolive (3D Cell Explorer).

\section{Results and Discussion}

Patient G., a man, 35 years old, was on outpatient observation with a cutaneous form of mastocytosis. Patient $T$., a woman, 41 years old, had a diffuse cutaneous form of mastocytosis. Hyperpigmentation of basal layer cells, mild epidermal edema and focal hyperkeratosis with loosening of the horny masses due to edema were detected in histological preparations of the skin in patients. Vacuolization was observed in individual cells of the stratum basale and the stratum spinosum (or the prickle cell layer) in the skin epidermis, that is a manifestation of vacuole hydropic dystrophy, characterized by intracellular edema of keratinocytes with the formation of vacuoles in their cytoplasm, which is an inducer of apoptosis. Individual pictures of acantholysis were identified. Immunohistochemical staining for LYVE-1 revealed that lymphatic vessels were located in the horizontal plane of the papillary layer of the skin dermis, often under the epidermis basal layer and they were wider than blood vessels. Then they plunged into a deeper dermis, where they formed a network. LYVE-1 positive staining was also detected in the sebaceous glands of the skin. In addition, tagging of skin for LYVE-1 revealed a pronounced positive signal for the marker in the cells of the stratum spinosum in the epidermis and a weaker signal for this studied marker in the cells of the epidermis stratum basale in patients with cutaneous mastocytosis. LYVE-1 is a hyaladherin, containing the Link domain - a protein capable of binding to hyaluronic acid. In humans it is encoded by the LYVE1 gene. LYVE-1 is a type I integral membrane glycoprotein. It binds to both soluble and immobilized hyaluronan. Our 3D modeling of the LYVE-1 marker expression in patients' skin epidermis revealed clear boundaries of prelimphatics and identified that intercellular edema was most pronounced in the stratum spinosum of the epidermis, that led to the loss of communications between epithelial cells in limited areas.

\section{ACKNOWLEDGMENT}

The study is supported by the budget project of the Research Institute of Clinical and Experimental Lymphology - branch of ICG SB RAS (No.0324-2019-0046C-02). This work involved the use of equipment from the Center for Genetic Resources of Laboratory Animal at the Institute of Cytology and Genetics, Siberian Branch, Russian Acadymy of Sciences.

\section{REFERENCES}

[1] J.P. Sundberg, C.H. Pratt, K.A. Silva, V.E. Kennedy, T.M. Stearns, B.A. Sundberg, L.E. King and H. HogenEsch, "Dermal lymphatic dilation in a mouse model of alopecia areata," Exp. Mol. Pathol., vol. 100, № 2, pp. 332-336, 2016.

[2] D.A. Zhdanov, General anatomy and physiology of lymphatic system. Moscow: Medgiz, 1952, 336p. (In Russian).

[3] C.H. Tripp, B. Haid, V. Flacher, M. Sixt, H. Peter, J. Farkas, R. Gschwentner, L. Sorokin, N. Romani and P. Stoitzner, "The lymph vessel network in mouse skin visualised with antibodies against the hyaluronan receptor LYVE-1,” Immunobiology, vol. 213, № 9-10, pp. $715-728,2008$ 